B Wiebe, C Fremerey, M Ehlen. Neonatology and Paediatric Critical Care, Children's Hospital Asklepios Klinik St. Augustin, St. Augustin, Germany

Introduction Congenital toxoplasmosis, a parasitic disease caused by the protozoon toxoplasma gondii is one of the classic intrauterine infections. The combination of hydrocephalus, retinochorioditis, intracranial calcification and parenchymal necrosis is rare. The majority of infected newborns present with uncharacteristic symptoms or remain asymptomatic. We report on a newborn with congenital toxoplasmosis and extensive cerebral findings.

Case Report Term male newborn, pregnancy and delivery uneventful, no maternal toxoplasmosis screening, admission on day 18 due to lethargy, sucking and muscular weakness, increase in head circumference of $4 \mathrm{~cm}$ since birth with gaping cranial sutures and recurrent cerebral convulsions. Brain ultrasound demonstrated distinctive ventriculomegaly with multiple intraventricular filaments. Liquor findings revealed elevated protein and positive toxoplasmosis PCR. Serologically toxoplasmosa antibodies showed positive as well. Treatment was commenced with pyrimethamine, sulfadiazine and folin acid. Ophthamological examination showed microphthalmus and retinal scars; NMR reveals multiple necrosis of basal ganglia and cerebellum. Multiple neurosurgical interventions were indispensible due to progressive intraventricular filament formation and subdural hygroma. Furthermore, severe thrombosis of the vena cava superior and the subclavian veins developed due to extensive clotting activation. Catheter intervention to achieve recanalization was performed with subsequent enoxaprarin therapy. After development of sinus thrombosis and progressive intracranial haemorrhage intensive care treatment was limited. The baby deceased after 28 days of treatment.

Discussion Our case demonstrates that though maternal screening is available and despite of existing treatment options severe courses of the disease are still possible. In case of excessive increase in head circumference an elaborate search for intrauterine infections should be mandatory.

\section{PERINATAL (FETAL AND NEONATAL) DIAGNOSIS AND EVOLUTION OF CARDIAC TUMOURS}

doi:10.1136/archdischild-2012-302724.0540

${ }^{1} \mathrm{~L}$ Dimitiru, ${ }^{2} \mathrm{AG}$ Dimitiru, ${ }^{3} \mathrm{M}$ Stamatin. ${ }^{1}$ Pediatric Cardiology, Medex Medical Center; ${ }^{2}$ Pediatric Cardiology; ${ }^{N}$ Neonatology, University of Medicine and Pharmacy, lasi, Romania

Cardiac tumors are rarely symptomatic and highlighted in the fetus when the size and position do not interfere with intracardiac hemodynamic. Objectives. To present four cases of cardiac tumors, confirmed by Doppler echocardiography (Echo) performed in the first 14 days postnatal, 2 of which were already highlighted by fetal echocardiogtaphy. Cases presentation. Fetal echocardiography showed 3 and respetivelly 4 intracardiac mass, well circumscribed, oval, 6-12 mm diameter, with echogenic appearance increased from normal cardiac structure, located in the IVS and the posterior wall of the LV, slightly protrudes in the lumen but no significant obstruction of LV outflow tract. Postnatal Echo confirmed the fetal echocardiography diagnosis multiple cardiac rhabdomyama. ECG: no suggestive changes. Chest X-Ray: cardiomegaly. One of cases was later diagnosed with tuberous sclerosis Bourneville. Fetal echocardiography not extracardiac changes detected in this case. The three cases of rhabdomyoma evolved according to age, without major cardiac distress and while echocardiography showed mild involution of tumors size without complete disappearance In the fourth case, Echo in the neonatal period revealed atrioventricular septal defect with intracardiac masses, 2 of 3 pedicled, non obstructive, pleading for a multiple cardiac fibroma. Not cardiac arrhythmias were detected fetal and postnatal development. Conclusions. fetal ultrasound screening and especially at older age of pregnancy may reveal the presence of cardiac tumors, mainly rhabdomyoama, then confirmed by Echo postnatal. Monitoring these tumors both in utero and post natal to allow early detection of obstructive disorders, with sometimes severe cardiac distress and requiring cardiovascular surgery.

\section{THE BIOMECHANICAL EVALUATION OF GAIT IN MONITORING OF TREATMENT IN CHILDREN WITH CEREBRAL PALSY-PRELIMINARY DATA}

doi:10.1136/archdischild-2012-302724.0541

${ }^{1}$ IA Kopyta, ${ }^{2} \mathrm{R}$ Michnik, ${ }^{2} \mathrm{~K}$ Jochymczyk-Woźniak. 'Department of Neuropediatrics, Medical University of Silesia, Katowice; ${ }^{2}$ Department of Biomechatronics, Silesian University of Technology, Gliwice, Poland

The methods enable the objective gait evaluation of the gait in children with cerebral palsy (CP). One of the indexes used to the analysis is Gillette Gait Index (GGI), complying 16 clinically meaningful kinematic and three-dimensional parameters. The study was conducted with application of the three-dimensional system of gait analysis BTS Smart. Spatial-time parameters of gait and courses of angles of joint of lower limbs were determined on the basis of conducted research. Those parameters were used in estimation of Gillette Gait Index. The analyzed group consisted of 12 cerebral palsy children at the age of $5-13$ years. All the children were assessed by the team before and after the botulin injection. The authors present the result of on the base of one of one patient, a girl at the age of 12 with right-sided spastic hemiparesis. The patient was evaluated three-times: before the botulin treatment, then three and six months after botulin injection. The value of the mean antetorsion of the pelvis in the saggital plain is now comparable to the healthy children at the same age. The GGI index improved in both lower extremities, for the right one within $24.14 \%$ and for the left one up to $40.69 \%$. The authors regard the results presented above as the pilot-study; the evaluation of the larger groups of children with cerebral palsy is being planned. In the authors 'opinion the objective method of CP children gait evaluation may be the helpful tool for clinicians to optimize the way of CP children treatment.

\section{A RARE CAUSE OF SWALLOWING DIFFICULTY THAT SHOULD BE ALWAYS IN MIND}

doi:10.1136/archdischild-2012-302724.0542

'M Ugras, 'S Bicer, ${ }^{2} \mathrm{~N}$ Tasdelen, 'A Vitrinel. 'Pediatrics; ${ }^{2}$ Radiology, Yeditepe Univ Medical Faculty, Istanbul, Turkey

Aim Swallowing difficulty among children is not a frequent complaint. Generally this problem is associated with gastroesophageal reflux, esophagitis, and rarely is seen as an outcome of esophageal strictures. We report here a boy who was admitted to our clinic with swallowing difficulty but finally had a different diagnose.

Material and Method A 6-year-old boy was admitted due to swallowing difficulty. The family was in France and the child had this complaint almost for 4 months. The boy had lost $5 \mathrm{~kg}$ but still was in the 50th centile both for weight and height. His physical, neurological and fundoscopic examinations were normal. But he was in a bad mood and seemed exhausted. He told that he could not swallow large particles and his portions became smaller gradually. Esophageal narrowing/stricture was thought thus Barium enema was performed and showed a normal esophagus. Then upper gastrointestinal endoscopy was performed, Endoscopical esophagitis and gastritis was confirmed by pathology with $\mathrm{H}$. pylori gastritis. Although the boy was put on PPI and antibiotics his complaints did not improve, and he was still in a depressed mood. Finally a cranial MR was taken and showed a 